\title{
Exploring Energy Aware Clustering Protocol for Heterogeneous Sensor Network
}

\author{
Shashi Kant Gupta \\ NIMS, University, Jaipur
}

\author{
SaurabhShrivastava,Ph.D \\ Bundelkhand University, Jhansi
}

\begin{abstract}
Wireless sensor network is a promising technology that plays a vital role in various applications i.e. disaster management, battle field surveillance and monitoring applications. It is especially useful in catastrophic or emergency scenario where human participation may be too dangerous. The sensor networks have evolved over a period of time. The failures are inevitable in wireless sensor networks due to inhospitable environment and unattended deployment; therefore sensor nodes must operate potentially in large numbers. The latest generation of sensors encompasses self organizing, flexible and scalable networks. The major constraints of wireless sensor network are the energy optimization. Heterogeneous sensor network work effectively to prolong the life time of the sensor network. In this paper we study on Energy Aware clustering protocol for Heterogeneous sensor network (EACP) and through simulation we analyze the impact of sensor network and compare the result with stable election protocol and Energy Efficient Heterogeneous Clustered Scheme protocol.
\end{abstract}

\section{Keywords}

Heterogeneous sensor network, Clustering, Energy Aware Clustering Protocol

\section{INTRODUCTION}

Wireless Sensor Networks (WSNs) [1] have emerged as research areas with an overwhelming effect on practical application developments. The sensor nodes consist of sensing, data processing and communicating components. They can be used for continuous sensing, event detection as well as identification, location sensing and control of actuators. The nodes are deployed either inside the phenomenon or very close to it and can operate unattended. They can use their processing abilities to locally carry out simple computations and transmit only required and partially processed data. They may be organized into clusters or collaborate together to complete a task that is issued by the users.

Clustering is a useful technique to organize the sensors. Idea to mark a group of nodes i.e. cluster of nodes according to some rules is called clustering and the controller of that group is referred as cluster head. Cluster head received data from its cluster members, aggregates this data and transmit to the base station. Clustering effectively minimizes the energy consumption and reduces number of messages communicated to base station. Clustering technique will increase the network lifetime as well as reduces the complexity. In figure 1 shows the clustering approach for wireless sensor network. In which there are three clusters, each cluster head communicate to the base station.

Wireless Sensor network having major issue is the energy constraints. There are various methods are provided by many authors to effective use of energy. Most of the protocol or methods are based on homogeneous sensor network.

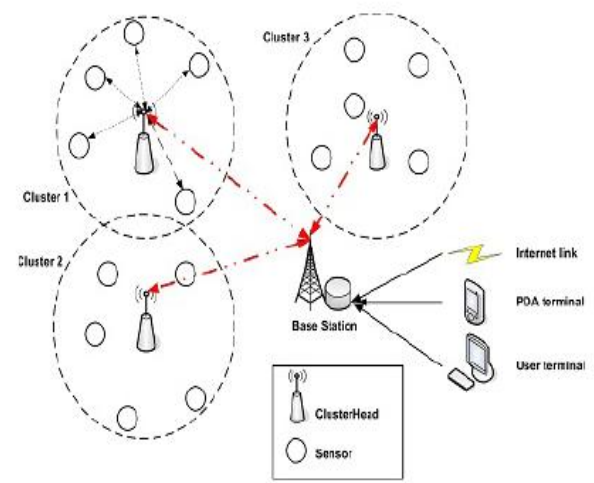

Fig.1 Clustering Structure for Wireless Sensor Network

Homogeneous sensor network is collection of inter related sensors having the same capability. Capability means sensors having same memory, energy and same processing. LEACH and HEED are some popular energy efficient protocol.

In recent year some protocol i.e. Stable Election Protocol (SEP) and Energy Efficient Heterogeneous Cluster Protocol (EEHC) is some popular protocol that have taken the advantages of heterogeneity. Heterogeneous sensor network is the collection of sensors network having different capability.

\section{HETEROGENEOUS SENSOR NETWORK}

In wireless sensor network heterogeneity defined as different in some aspects. Heterogeneity plays an important in $\mathrm{n}$ real life applications i.e. for monitoring and automation applications in home like environments and industrial security

\subsection{Heterogeneous Resources}

There are three types of heterogeneity [2].

- Link Heterogeneity: This type of heterogeneous node having long distance highly reliable communication node. It reduces the average number of hops that data packets take from sensor node to the base station. Link Heterogeneity provide bypass highway across the network concurrently increasing end to end delivery rate and decreasing energy consumption.

- Computation Heterogeneity : Nodes having more computational power in compare to other nodes

- Energy Heterogeneity: Nodes have unlimited or higher energy resources. 


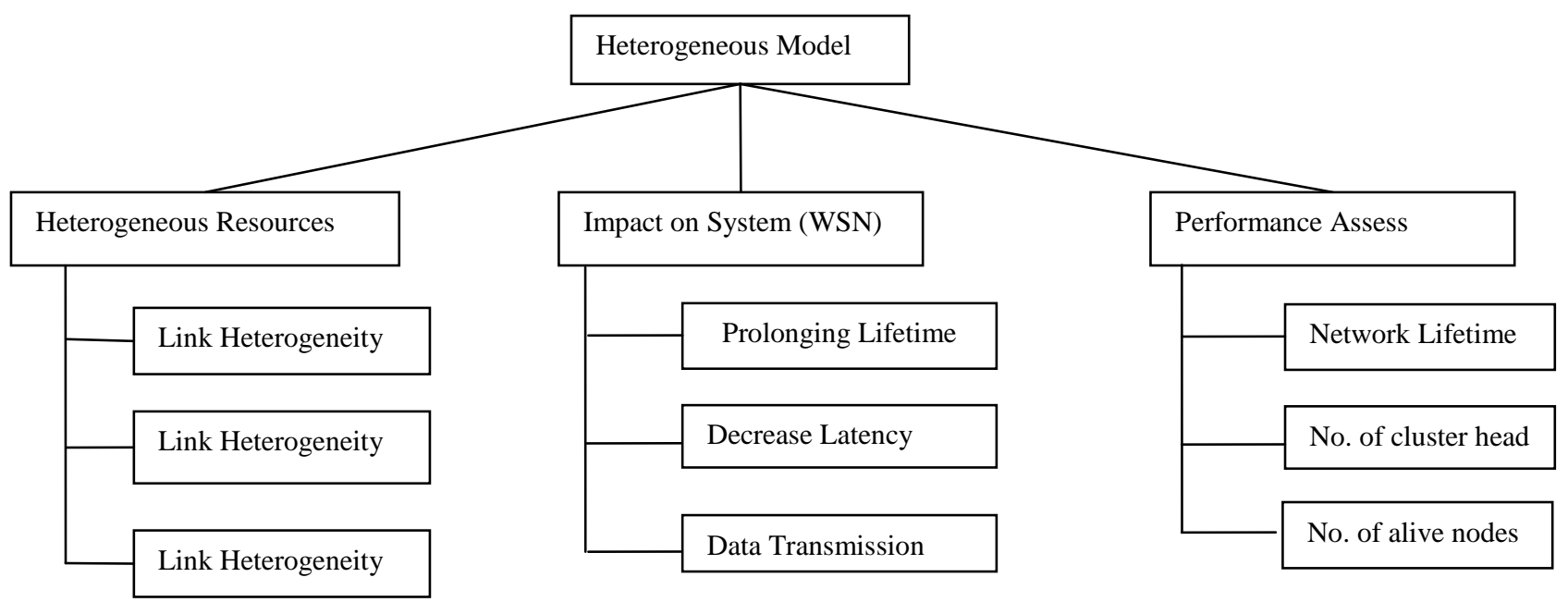

Fig.2: Heterogeneous Sensor Model

\subsection{Impact on System}

Heterogeneity having great impact on sensor network. Some of the advantages are listed below

Prolonging Lifetime: If we organize sensor properly i.e. we elect the higher energy node as cluster head, it will increase the life time of the system.

Decrease Latency: Computational heterogeneity decreases the computational latency and link heterogeneity will decreases waiting time in the transmission of data.

Data Transmission: One of the issues with wireless sensor network is low reliability of links. With the heterogeneous sensor network provide the reliable link by minimizing the hops between the node and the Base station.

\subsection{Performance Assess}

To evaluate the performance of heterogeneous sensor network protocol some of the assessment parameter are used

Network Lifetime: It is defined in two ways, some of the researchers says "it is the time interval from start of the operation to the die of the first node of the sensor network" Some says start of the operation to the die of last node of the sensor network. We use first one.

No. of Cluster Head: How many numbers of clusters are elected in each round. The number of cluster head reflects the energy consumption.

No. of alive node: it is number of alive node in sensor network in each round.

\section{RELATED WORK}

In recent years, energy consumption in wireless sensor network has been investigated widely. Energy consumption can be worked in form of cluster size [3], efficient number of hop in network [4], optimal transmission algorithm [5] or routing solutions

Low Energy Adaptive Clustering Protocol (LEACH) is developed by Heinemann [6], LEACH is the $t$ most clustering popular energy efficient protocol for homogeneous sensor network. LEACH having two phases

- Setup Phase: In this phase cluster-heads are chosen

- Steady Phase: The cluster-head is maintained when data is transmitted between sensors.
LEACH is a probabilistic protocol in which cluster head is selected randomly and provide the aggregation through which reduces the number of transmission.

In setup phase some part of node $p$, elect themselves as cluster heads. A sensor node selects a random number $r$. The value of $r$ must be between 0 and 1 . The election of node whether it is cluster head or work as normal node depends on the value of threshold $\mathrm{T}(\mathrm{n})$. If the random number $\mathrm{r}$ is less then $\mathrm{T}(\mathrm{n})$ then it work as cluster head.

The problem with LEACH is Selecting cluster heads is favourable in the beginning but unfavourable in later rounds and motive of data transmission is lost while nodes are busy with cluster head selection.

A modified version of LEACH-C is introduced by M. Younis, M. Youssef, K. Arisha [11], is a centralized cluster formation version of $\mathrm{LEACH}$, where the base station organizes and controls the network. More precisely, LEACH-C protocol provides a centralized cluster formation, local processing for aggregation of sensed data and the rotation of $\mathrm{CHs}$ for every round. These activities are aimed at achieving uniform energy consumption among sensor nodes and maximizing network lifetime. Since, the BS does not have energy constraint, centralized cluster formation methods can be attractive alternatives.

Hybrid Energy Efficient Distributed Clustering Approach (HEED) is introduced by Ossama Younis and Sonia Fahmy [8]. It is work on residual energy of the node. HEED prolong the life time of the network and minimizing the control overhead. HEED protocol used two parameters

(i) Probabilistically take an initial set of cluster heads.

(ii) "Break ties" among cluster heads. A tie means a node falls within the "range" of more than one cluster head.

In first phase of algorithm sets the initial percentage of cluster head. The percentage of cluster head $\mathrm{CH}_{\text {prop }}$ is defined by

$$
\mathrm{CH}_{\text {prob }}=C_{\text {prob }} \times \frac{E_{\text {residual }}}{E_{\text {max }}}
$$

$\mathrm{E}_{\text {residual }}$ is the current energy of node and $\mathrm{E}_{\max }$ is the maximum energy corresponds to a fully charged battery of node. The value of $\mathrm{CH}_{\text {prob }}$ is not allowed to fall below the threshold.

Stable Election Protocol (SEP) is develop for heterogeneous sensor network [9]. It is improvement over LEACH. SEP work on the principal of advance nodes has become cluster head more often than the normal nodes. SEP is worked on the 
principle of weighted election probability of each node to become cluster head according to the remaining energy in each node. Eo is the initial energy of each normal sensor. The energy of each advanced node will be Eo $\cdot(1+\alpha)$. The total energy of the new heterogeneous setting is equal to

$$
T=n * E o *(1+\alpha * \mathrm{~m})
$$

the total energy of the system is increased by $1+\alpha \cdot \mathrm{m}$ times.

The weighed probabilities for normal and advanced nodes are, respectively:

$$
\begin{gathered}
\text { Pnrm }=\frac{\text { Popt }}{1+\alpha \cdot m} \\
\text { Padv }=\frac{\text { Popt }}{1+\alpha \cdot \mathrm{m}} \times(1+\alpha)
\end{gathered}
$$

The thresh hold value for normal and advance node will be calculated as

$$
\begin{aligned}
& T(\text { Snrm })=\frac{\text { Pnrm }}{1-\text { Pnrm } *\left(\mathrm{r} \text { mod } \frac{1}{\text { Prrm }}\right)} \text { if } S_{\text {nrm }} € \text { G' otherwise } 0 \\
& T(\operatorname{Sadv})=\frac{\text { Padv }}{1-\text { Padv } *\left(\mathrm{r} \text { mod } \frac{1}{\text { Padv }}\right)} \text { if } S_{\text {adv }} € \text { G', otherwise } 0
\end{aligned}
$$

where $r$ is the current round, G' is the set of nodes that have not become cluster heads within the last $1 / \mathrm{P}_{\mathrm{nrm}}$ rounds and $\mathrm{T}\left(\mathrm{S}_{\mathrm{nrm}}\right)$ is the thresh hold value. G' ${ }^{\prime}$ is the set of nodes that have not become cluster heads within the last $1 / \mathrm{P}_{\mathrm{adv}}$ rounds and $T\left(S_{a d v}\right)$ is the thresh hold value. SEP enhances the stability period of the network.

Dilip Kumara, Trilok C. Aseri and R.B. Patel proposed Energy efficient heterogeneous clustered scheme for wireless sensor networks (EEHC) [12] based on weighted election probabilities of each node to become a cluster head according to the residual energy in each node. $\mathrm{CHs}$ collected the sensors' readings in their individual clusters and send an aggregated report to the base-station.

\section{ENERGY AWARE CLUSTERING PROTOCOL FOR HETEROGENEOUS SENSOR NETWORK}

EACP worked on heterogeneous sensor network were sensor had different energy heterogeneity and rest of the heterogeneity is same for all nodes. In such network some nodes having power supply by main line some are powered by AAA batteries or AA battery.

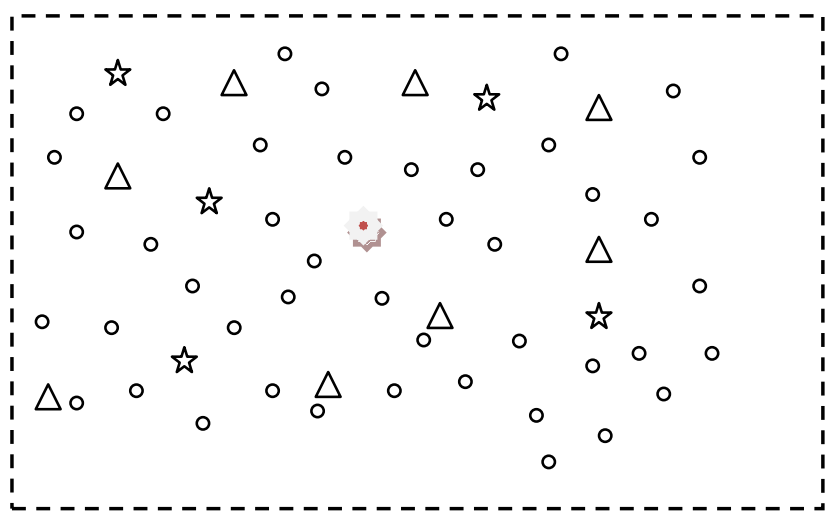

Fig.3 Heterogeneous Sensor Structure

Figure 3 shows the randomized distribution of sensor nodes having different energy. Stars are powered by mains, triangles are powered by AAA battery and circle powered by AA battery. Red circle is the base station of the network. EACP design an optical way by considering following parameters.

- Data Packet Size

- Frequency of Transmission

- Local Computation Process

- $\quad$ Minimizing delay

Energy Aware Clustering Protocol worked on following phases

- Reporting Phase

- Clustering Phase

- Load Balancing

- Data Transmission Phase

\subsection{Reporting Phase:}

In this phase all the sensor send their initial energy to the base station through the packet, which contents the address of sender, receiver and the initial energy of the node.

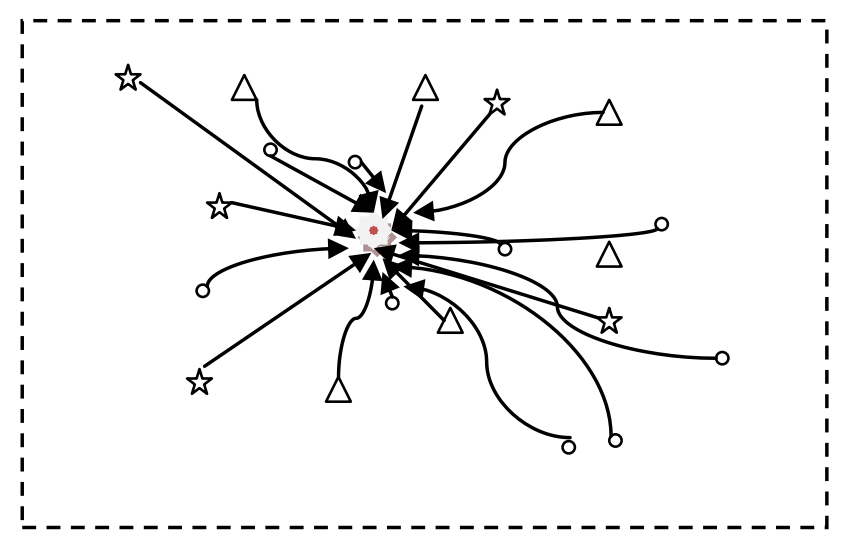

Fig. Single hop communication

Reporting phase uses single hop communication. It consume more energy than multi-hop communication due to transmission power is proportional to $\mathrm{d}^{\mathrm{n}}$ were $\mathrm{d}$ is distance between node. Node which is far from base station more energy is wasted as compare to nearer node.

We improvised it by using the multi hop communication and by simulation we show it save $40 \%$ energy as compare to SEP and EEHC protocol.

\subsection{Clustering Phase:}

In clustering phase Base station elect the cluster head on the basis of initial energy of the node. After elected node as a cluster head, the node will send a message to all other nodes "I am the cluster head" on the basis of strength of signal other nodes will decide whether they want to become member node of the cluster head by sending acknowledgement with their current energy.

In Fig4 shows there are four clusters have made and star sign indicate as cluster head and having responsibility to aggregate the data and send to the base station. 


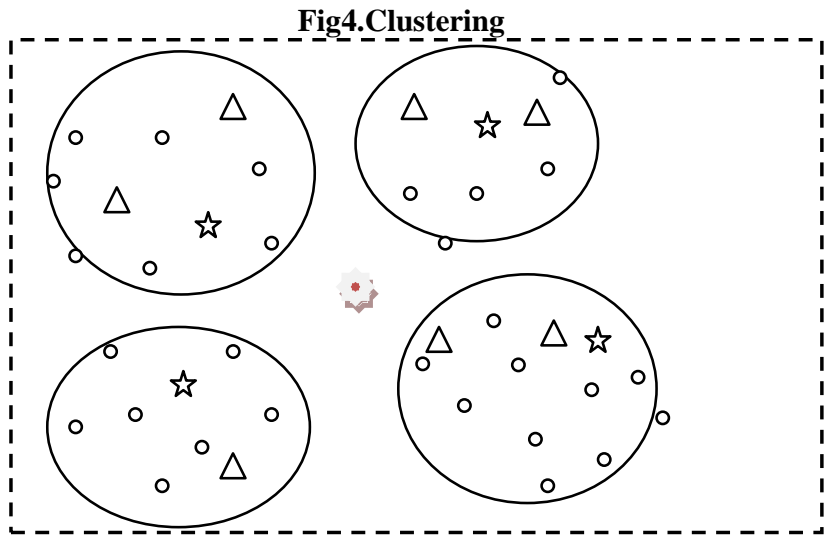

\subsection{Load Balancing Phase:}

In this clustering technique some cluster may have huge no of member node and some have lesser number. It creates load unbalancing to overcome of this problem EACP introduce secondary cluster head.

Selection of secondary cluster head is based on current energy of member nodes. This record is maintained by the cluster head. Cluster head distribute pass there load in two cases

1. Whenever the current energy of the cluster head has reduced by $50 \%$ of its current energy.

2. Processing delay occur at cluster head.

\subsection{DATA TRANSMISSION PHASE:}

In this phase aggregate data will transmit to the cluster head to base station by multi-hop communication. To send the packet to the base station there should be an optimal path to send the data packet. The path should be shortest and minimum delay to send the packet. EACP uses OSPF (Open Shortest Path First) to send the data packet to Base Station. EACP uses first order radio model for communication [9]

\section{SIMULATION RESULT:}

We have simulated result of EACP protocol and compare the result EEHC and SEP protocol and find some useful result. We use NS2 simulator and following parameter used

\begin{tabular}{|l|l|}
\hline Area & $100^{*} 100 \mathrm{~m}$ \\
\hline No. of node & 50 \\
\hline $\begin{array}{l}\text { Initial Energy of those nodes powered by A } \\
\text { battery }\end{array}$ & $.5 \mathrm{~J}$ \\
\hline $\begin{array}{l}\text { Initial Energy of those nodes powered by } \\
\text { AA battery }\end{array}$ & $.1 \mathrm{~J}$ \\
\hline Transmitter Amplifier Energy Deception & $5 * 10^{\wedge}-9 \mathrm{~J}$ \\
ETx (Transmit) & $5 * 10^{\wedge}-9 \mathrm{~J}$ \\
\hline ERx (Received) & $5^{*} 10^{\wedge}-9 \mathrm{~J}$ \\
\hline Date Aggregation Energy & 1000 bits \\
\hline Packet Size & \\
\hline
\end{tabular}

We found some interesting result for life time sensor network. In order to investigate energy efficiency with life time extension, the performance of EACP protocols are evaluated through NS2 simulator, which shows the energy efficiency and lifetime of the network

\begin{tabular}{|l|l|l|l|}
\hline Protocol & FND & HNA & LND \\
\hline SEP & 134 & 320 & 720 \\
\hline EEHC & 142 & 270 & 673 \\
\hline EACP & 70 & 570 & - \\
\hline
\end{tabular}

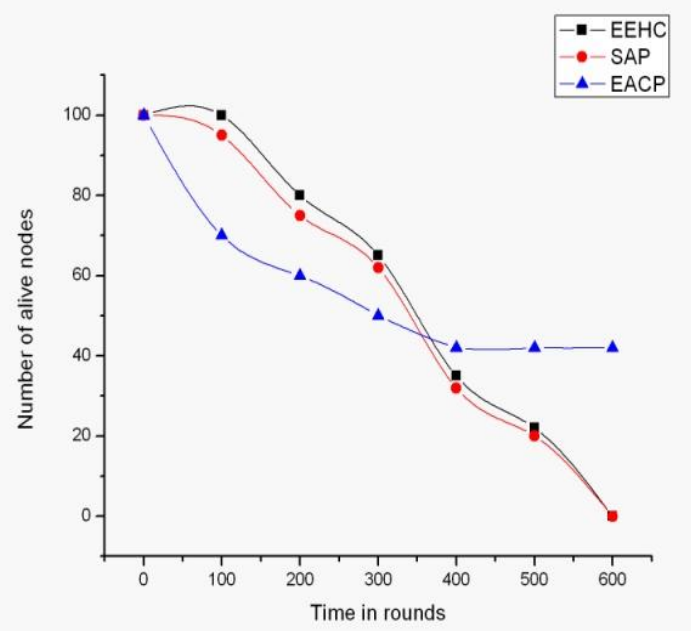

Fig5

As table 2 shows at node never die because in design of our structure some nodes are directly connected with main (unlimited power supply) so that these nodes cannot die. In stable election protocol first node will die at 134 rounds and EEHC protocol first node will die at 142 rounds but in EACP first node will die at 70 . It is because in reporting phase all nodes send their initial energy to base station in this duration energy has drain out quickly as well as in cluster head selection some energy has wasted.

Figure 5 shows EACP protocol having higher life time and save up $40 \%$ more energy as compare to SAP and EEHC.

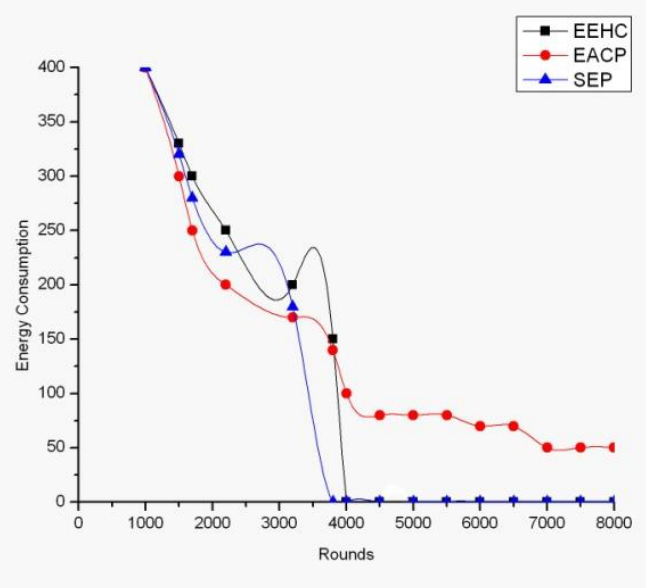

Fig6.

Fig6 shows the energy consumption rate in respect to rounds simulation shows that EACP protocol initially decreases very fast due to find the those nodes having higher energy or 
connected with mains. In compare to SEP and EEHC protocol initially energy consumption rate is lower compare to EACP but later on EACP protocol will stable as mains node has found.

\section{CONCLUSION}

Energy Aware Clustering Protocol is application specific protocol and produces better result in terms of lifetime of sensor network for heterogeneous sensor network. It is effectively selecting the cluster head and using secondary cluster head selection it balance the load effectively. Security is the important issue for home sensor network. Any good security model for heterogeneous sensor network will fit for this protocol. Such security model is our next future direction

\section{REFERENCES}

[1] Q. Wang, H.S. Hassanein and K. Xu, "A practical perspective on wireless sensor networks", Chapter 9, Handbook of Sensor Networks: Compact Wireless and Wired Sensing Systems (Edited By Mohammad Ilyas abnd Imad Mahgoub), CRC Press, 2005. ISBN 0-84931968-4

[2] Razieh , Sam Jabbehdari , Ahmad Khadem 2011, Comparison of Energy Effeicent Clustering Protocol in Heterogeneous Wirless Sensor Network in IJAST vol.36

[3] N. Kim, S. Han, W.H. Kwon, Optimizing the number of clusters in multi-hop wireless sensor networks, IEICE Transactions on Communications E91-B (1) (2008) 318321Tavel, P. 2007 Modeling and Simulation Design. AK Peters Ltd.

[4] T. Shu, M. Krunz, S. Vrudhula, Power balanced coverage-time optimization for clustered wireless sensor networks, in: Proceedings of the MobiHoc, 2005, pp. 111-120.

[5] A. Manjeshwar and D. P. Agrawal; TEEN: A routing protocol for enhanced efficiency in wireless sensor networks. Proceedings of the IPDPS Workshop on Issues in Wireless Networks and Mobile Computing. San Francisco, CA, Apr. 2001, pp.2009- 2015. MA, Nov. 2000, pp.63-73
[6] W. Heinzelman, A. Chandrakasan and H. Balakarishnan, "Energy-Efficient Communication Protocols for Wireless Microsensor Networks," Proceedings of the Hawaaian International Conference on Systems Science, January 2000.

[7] Heinzelman, W. R., A. Chandrakasan, and H. Balakrishnan, "An application-specific protocol architecture for wireless micro sensor networks," IEEE Tran.on Wireless Comm., Vol. 1, No. 4, 660- 670, Oct. 2002.

[8] Ossama Younis and Sonia Fahmy "HEED: A Hybrid, Energy-Efficient, Distributed Clustering Approach for Ad-hoc Sensor Networks" IEEE Transactions on Mobile Computing, 3(4): 366-379, 2004.

[9] G. Smaragdakis, I. Matta, A. Bestavros, SEP: A Stable Election Protocol for clustered heterogeneous wireless sensor networks, in: Second International Workshop on Sensor and Actor Network Protocols and Applications (SANPA 2004), 2004.

[10] W. R. Heinzelman, A. P. Chandrakasan, and H. Balakrishnan., "An application-specific protocol architecture for wireless microsensor networks. IEEE Transactions on Wireless Communications", 1(4):660 670, October 2002

[11] M. Younis, M. Youssef, K. Arisha, Energy-aware management in cluster-based sensor networks, Computer Networks 43 (5) (2003) 649-668.

[12] D. Kumar, T. C. Aseri, and R. B. Patel, "EEHC: Energy Efficient Hetererogeneous Clustered Scheme forWireless Sensor Networks," Computer Communications, 32(4): 662-667, March 2009. 